\title{
ACIDENTES DE TRABALHO COM MATERIAIS BIOLÓGICOS ENTRE PROFISSIONAIS DE ENFERMAGEM: uma revisão integrativa
}

\author{
Ann Gracielle Moreira GOMES \\ Tatianne da Costa SABINO2 \\ Rosangela Vida de NEGREIROS ${ }^{3}$
}

\begin{abstract}
${ }^{1}$ Especialista em Enfermagem do trabalho, enfermeira assistencial do Hospital de Universitário Alcides Carneiro. Email: anngracielle@ hotmail.com.br.

${ }^{2}$ Acadêmica de Enfermagem da Universidade Federal de Campina Grande, Campina Grande-PB. Emai: taty_sabino94@hotmail.com

${ }^{3}$ Mestre em Enfermagem. Docente do Curso de Enfermagem da Universidade Federal de Campina Grande. Email: rosangelavn@ufcg.edu.br.
\end{abstract}

Recebido em: 21/12/2015 - Aprovado em: 0711/2016 - Disponibilizado em: 18/12/2016

\begin{abstract}
RESUMO:
O presente estudo trata-se de uma revisão integrativa de literatura que teve como objetivo analisar as produções científicas existentes sobre acidentes de trabalho com materiais biológicos dos profissionais de Enfermagem entre os anos de 2009 a 2015. Metodologia: A pesquisa foi realizada do período de junho de 2014 a dezembro de 2015. Nas bases de dados BDENF e LILACS, foram utilizados os descritores "Acidentes de trabalho", "Enfermagem", "Hospital", "Saúde do Trabalhador" e "Trabalhador", conforme os descritores em Ciências da Saúde da Biblioteca Virtual em Saúde, no qual, foram selecionados 10 artigos. Resultados e Discussões: De acordo com os artigos analisados pode-se constatar que a classe de Enfermagem mais exposta aos riscos biológicos é auxiliar de enfermagem, por ser responsável a procedimentos mais invasivos e utilizarem materiais perfuro cortantes rotineiramente. Os aspectos psicológicos também foram abordados como os sentimentos de tristeza, ansiedade, angústia e desespero em que os profissionais passam ao serem acidentados. As subnotificações são muito comuns e limitam o desempenho das tomadas de decisões e na qualidade de prestação de serviço ao acidentado. Conclusão: É de fundamental importância ações de educação permanente acerca da adoção das estratégias de biossegurança com ênfase na saúde e qualidade de vida do trabalhador, no âmbito de suas atividades laborais. Além do fornecimento de materiais mais seguros e mudança nas condições de trabalho dos profissionais de enfermagem.
\end{abstract}

Palavras- chave: Acidentes de Trabalho. Enfermagem. Hospital. Saúde do Trabalhador. Trabalhador.

\begin{abstract}
:
This study deals with an integrative literature review that aimed to analyze the existing scientific production on occupational accidents with biological materials of nursing professionals between the years 2009 to 2015 . Methodology: The survey was conducted in the period June 2014 to December 2015. In the databases BDENF and LILACS, the keywords "Occupational Accidents" were used, "Nursing", "Hospital", "Occupational Health" and "worker" as descriptors in Health Sciences Virtual Health Library, which were selected 10 articles. Results and Discussion: According to the articles analyzed it can be seen that the most exposed to biological hazards Nursing class is nursing assistant, to be responsible for the more invasive procedures and use sharp punch materials routinely. The psychological aspects were also addressed such as feelings of sadness, anxiety, anguish and despair in which professionals spend to be rough. Underreporting is common and limit the performance of decision-making and quality of service to the injured. Conclusion: It is vital continuing education activities about the adoption of biosecurity strategies with emphasis on worker health and quality of life, as part of their work activities. In addition to providing the safest and change in working conditions for nurses materials.
\end{abstract}

Keywords: Occupational Accidents .Nursing. Hospital. Occupational Health.Worker. 


\section{INTRODUÇÃO}

O trabalho em saúde é fundamental para vida humana, por ser uma ação transformadora relacionada aos sujeitos que recebem a assistência, além da íntima relação entre o processo de produção e o produto do trabalho (PIRES, 2008). Entretanto, nem sempre no trabalho existem condições favoráveis à saúde do trabalhador.

O Acidente de Trabalho é aquele que ocorre durante o exercício da profissão e consiste em lesão corporal ou perturbação funcional, com perda ou redução da capacidade para o trabalho, de forma permanente ou temporária, ou até mesmo a morte (VALIM; MARZIALE, 2011).

Os acidentes e as mortes no trabalho estão entre as maiores problemáticas relacionadas à saúde do trabalhador no Brasil. O Ministério da Saúde tem elaborado estratégias na tentativa de reduzir essa situação, como a implantação da Política Nacional de Notificação de Acidentes e Doenças no Trabalho que tem resultado na criação da Rede Nacional de Atenção à Saúde do Trabalhador - RENAST e os Centros de Referência em Saúde do Trabalhador CEREST em todo o País, desde o ano de 2000 (PROCHNOW; MAGNAGO, et al. 2012).

Os acidentes de trabalho constituem um importante problema de saúde pública em todo o mundo. As estimativas da Organização
Internacional do Trabalho revelam a ocorrência anual de 160 milhões de doenças ocupacionais, 250 milhões de acidentes de trabalho e 330 mil óbitos, baseando-se somente em doenças não transmissíveis. Embora acidentes com material biológico não se enquadrem na definição legal de acidente de trabalho, o registro junto à Medicina do Trabalho e Comissão de Controle de Infecção Hospitalar - $\mathrm{CCIH}$ é fundamental, considerando-se suas consequências (SIMÃO et al. , 2010).

O risco biológico é proveniente do contato dos trabalhadores com microorganismos, sobretudo vírus e bactérias, que podem levar a aquisição de doenças como a Síndrome da Imunodeficiência Adquirida AIDS e Hepatites. $\mathrm{Na}$ enfermagem a exposição da equipe a cargas biológicas advém da manipulação de pacientes com doenças transmissíveis e infectocontagiosas, ferida cirúrgica, desinfecção e esterilização de materiais contaminados, entre outros. Como agravantes, a falta de Equipamentos de Proteção Individual - EPI em quantidade e qualidade adequadas e a ausência de treinamento influenciam na ocorrência de acidente de trabalho (RIBEIRO, 2007).

As condições de trabalho da equipe de enfermagem, principalmente nos hospitais, têm sido consideradas impróprias no que concerne às especificidades do ambiente gerador de riscos à saúde (SCHMOELLER et 
al., 2011). Esse profissional no âmbito hospitalar exerce uma diversidade de serviços, podendo desencadear situações de riscos, agredindo a sua saúde e refletindo na qualidade da assistência prestada.

Conforme as estatísticas observadas, a equipe de enfermagem é uma das principais categorias profissionais sujeitas às exposições com material biológico. Este fato relaciona-se à Enfermagem ser a profissão da área da saúde a ter mais contato direto na assistência aos pacientes e também ao tipo e à frequência de procedimentos realizados (SIMÃO et al., 2010).

Sendo assim, este estudo tem como objetivo analisar as produções científicas existentes acerca dos materiais biológicos envolvendo os acidentes de trabalho nos profissionais de enfermagem.

\section{METODOLOGIA}

O presente estudo se caracteriza como uma revisão integrativa realizada entre junho de 2014 a novembro de 2015, que buscou apresentar a revisão e análise crítica acerca do risco ocupacional biológico envolvendo o Acidente de trabalho entre profissionais de enfermagem. Este método de pesquisa consiste na construção de uma vasta análise da literatura, contribuindo para discussões sobre métodos e resultados de pesquisas, além de reflexões sobre a realização de futuros estudos. (BAKKE; ARAÚJO, 2010).

Para a elaboração as seguintes etapas foram realizadas: estabelecimento da hipótese, objetivos da revisão, critérios de inclusão e discussão dos resultados. Na coleta dos dados foram realizadas buscas bibliográficas no banco de dados da Literatura Latino-Americana e do Caribe em Ciências da Saúde - LILACS e Base de Dados de Enfermagem - BDENF. Os descritores foram cruzados e utilizados com o operador booleano AND: "Acidentes de Trabalho"; "Enfermagem"; "Saúde do trabalhador", "Hospital" e "Risco Biológico".

Os critérios de inclusão foram os artigos que obedecessem aos seguintes critérios: estudos que fazem abordagem sobre acidentes de trabalhos entre profissionais de enfermagem envolvendo materiais biológicos, publicações nacionais, publicados entres 2009 a 2014, divulgados na língua portuguesa e estudos disponibilizados na íntegra. Entretanto, os critérios de exclusão foram: dissertações, monografias, artigos que não estavam publicados em português, os que se repetiram e artigos que não estavam disponibilizados na íntegra.

Desta maneira, ao fazer a filtragem na base de dados Biblioteca Virtual em Saúde foram selecionados 26 artigos, ao escolher a base de dados LILACS, o cruzamento dos descritores resultaram em 15 artigos, porém 
apenas cinco foram inclusos, 11 no BDENF, nos quais foi selecionado cinco, constituindo assim uma amostra de 10 artigos.

\section{RESULTADOS E DISCUSSÃO}

Após categorização dos artigos abordagens, produzidas no campo de acidentes de trabalho envolvendo os profissionais de enfermagem. A partir da constatação, foram reunidos os resultados encontrados em um modelo de fácil compreensão para uma melhor síntese dos assuntos focados.

selecionados, foi possível observar as diversas

Quadro 1- Apresentação dos artigos, considerando os autores, ano de publicação, título do artigo, tipo de estudo e objetivos. 2015.

\begin{tabular}{|c|c|c|c|c|}
\hline AUTORES & $\begin{array}{c}\text { ANO DE } \\
\text { PUBLICAÇÃO }\end{array}$ & TÍTULO DO ARTIGO & $\begin{array}{l}\text { TIPO DE } \\
\text { ESTUDO }\end{array}$ & OBJETIVOS \\
\hline $\begin{array}{l}\text { LIMA, Lílian } \\
\text { Moura de; } \\
\text { OLIVEIRA, } \\
\text { Camila Cardoso } \\
\text { de; } \\
\text { RODRIGUES, } \\
\text { katiuscia Milano } \\
\text { Rosales de. }\end{array}$ & 2011 & 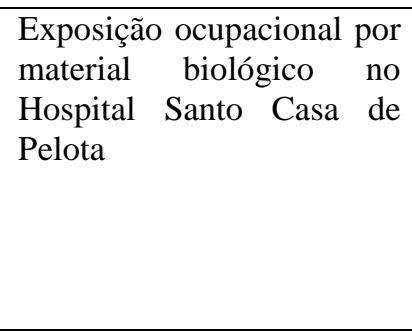 & $\begin{array}{l}\text { Abordagem } \\
\text { quantitativa } \\
\text { com } \\
\text { delineamento } \\
\text { transversal }\end{array}$ & $\begin{array}{l}\text { Caracterizar os acidentes de } \\
\text { trabalho que envolve material } \\
\text { biológico ocorrido no Hospital } \\
\text { Santo casa de Pelota }\end{array}$ \\
\hline $\begin{array}{l}\text { MARZIALE, } \\
\text { Maria Helena } \\
\text { Palucci et al. }\end{array}$ & 2014 & \begin{tabular}{l} 
Conseqüências $\quad \mathrm{da}$ \\
exposiçẫo ocupacional a \\
material biológico entre \\
trabalhadores de um \\
\multicolumn{2}{l}{ hospital universitário }
\end{tabular} & $\begin{array}{l}\text { Estudo } \\
\text { descritivo } \\
\text { exploratório } \\
\text { com } \\
\text { abordagem } \\
\text { Quantitativa }\end{array}$ & $\begin{array}{l}\text { Analisar a ocorrência as } \\
\text { características e consequiência do } \\
\text { acidente de trabalho com } \\
\text { exposição a material biológico } \\
\text { por trabalhadores e instituição }\end{array}$ \\
\hline $\begin{array}{l}\text { ARAÚJO, } \\
\text { Thiago Moura et } \\
\text { al. }\end{array}$ & 2012 & $\begin{array}{l}\text { Acidente ocupacional e } \\
\text { contaminação pelo HIV: } \\
\text { sentimentos vivenciados } \\
\text { pelos profissionais de } \\
\text { enfermagem }\end{array}$ & $\begin{array}{l}\text { Estudo } \\
\text { Descritivo com } \\
\text { abordagem } \\
\text { qualitativa }\end{array}$ & $\begin{array}{l}\text { Conhecer sentimentos e emoções } \\
\text { dos profissionais de Enfermagem } \\
\text { que sofreram acidentes } \\
\text { ocupacionais com exposição a } \\
\text { material biológico contaminado } \\
\text { pelo vírus HIV }\end{array}$ \\
\hline $\begin{array}{l}\text { RIBEIRO, } \\
\text { Luana Cássia } \\
\text { Miranda et al. }\end{array}$ & 2010 & $\begin{array}{l}\text { Influencia da exposição à } \\
\text { material biológico na } \\
\text { adesão ao uso de } \\
\text { equipamentos de proteção } \\
\text { individual }\end{array}$ & $\begin{array}{l}\text { Estudo } \\
\text { descritivo com } \\
\text { abordagem } \\
\text { Qualitativa }\end{array}$ & $\begin{array}{l}\text { Avaliar se a exposição a material } \\
\text { biológico é um fator } \\
\text { determinante para adesão ao uso } \\
\text { de equipamentos de proteção } \\
\text { individual por parte aos } \\
\text { trabalhadores de Enfermagem }\end{array}$ \\
\hline $\begin{array}{l}\text { GUILARD, } \\
\text { Adriana } \\
\text { Oliveira et al. }\end{array}$ & 2010 & $\begin{array}{lr}\text { Acidentes com } & \text { material } \\
\text { Biológico } & \text { entre } \\
\text { profissionais de } & \text { Hospital } \\
\text { Universitário em Goiânia. }\end{array}$ & $\begin{array}{l}\text { Estudo } \\
\text { Descritivo com } \\
\text { abordagem } \\
\text { quantitativa }\end{array}$ & $\begin{array}{llr}\text { Avaliar } & \text { os } & \text { acidentes } \\
\text { profissionais com material } \\
\text { biológicos ocorridos entre } \\
\text { profissionais do hospital das } \\
\text { clinicas da universidade federal } \\
\text { de Goiás }\end{array}$ \\
\hline $\begin{array}{l}\text { MACHADO, } \\
\text { Rosária } \\
\text { Mascaro; }\end{array}$ & 2011 & $\begin{array}{l}\text { Acidentes com material } \\
\text { biológico em trabalhadores } \\
\text { de Enfermagem do } \\
\text { Hospital Geral de Palmas }\end{array}$ & $\begin{array}{l}\text { Estudo } \\
\text { Descritivo com } \\
\text { delineamento } \\
\text { Transversal }\end{array}$ & $\begin{array}{l}\text { Identificar os acidentes de } \\
\text { trabalho com material biológico } \\
\text { ocorridos com os trabalhadores } \\
\text { de enfermagem do hospital geral }\end{array}$ \\
\hline
\end{tabular}




\begin{tabular}{|c|c|c|c|c|}
\hline $\begin{array}{l}\text { MACHADO, } \\
\text { Fernando de } \\
\text { Almeida et al. }\end{array}$ & & (TO) & $\begin{array}{l}\text { com } \\
\text { abordagem } \\
\text { quantitativa }\end{array}$ & $\begin{array}{l}\text { de Palmas e verificar a } \\
\text { ocorrência de subnotificações no } \\
\text { serviço de segurança do trabalho }\end{array}$ \\
\hline $\begin{array}{l}\text { GIANCOTTI, } \\
\text { Geanna } \\
\text { Mendonça et al. }\end{array}$ & 2014 & $\begin{array}{l}\text { Caracterização das vítimas } \\
\text { e dos acidentes de trabalho } \\
\text { com material biológico } \\
\text { atendidas em um hospital } \\
\text { público do Paraná }\end{array}$ & $\begin{array}{l}\text { Estudo } \\
\text { Descritivo com } \\
\text { abordagem } \\
\text { quantitativa }\end{array}$ & $\begin{array}{l}\text { Descrever as características dos } \\
\text { acidentes de trabalho com } \\
\text { material biológico atendidos em } \\
\text { um hospital público do Paraná }\end{array}$ \\
\hline $\begin{array}{l}\text { MAGAGNINI, } \\
\text { Maristela } \\
\text { Aparecida } \\
\text { Magri; } \\
\text { ROCHA, Suelen } \\
\text { Alves; AYRES, } \\
\text { Jairo Aparecido }\end{array}$ & 2011 & $\begin{array}{l}\text { O Significado do acidente } \\
\text { de trabalho com material } \\
\text { biológico para } \quad \text { os } \\
\text { profissionais } \\
\text { Enfermagem }\end{array}$ & $\begin{array}{l}\text { Delineamento } \\
\text { Transversal } \\
\text { com } \\
\text { abordagem } \\
\text { quantitativa }\end{array}$ & $\begin{array}{l}\text { Compreender o significado dos } \\
\text { acidentes de trabalho com } \\
\text { exposição a material biológico } \\
\text { nas perspectivas dos } \\
\text { profissionais de Enfermagem }\end{array}$ \\
\hline $\begin{array}{l}\text { SIMÃO, Suzana } \\
\text { de Almeida } \\
\text { Fráguas et al. }\end{array}$ & 2009 & $\begin{array}{lr}\text { Fatores associados } & \text { aos } \\
\text { acidentes biológicos } & \text { entre } \\
\text { profissionais } & \text { de } \\
\text { enfermagem } & \end{array}$ & $\begin{array}{l}\text { Estudo } \\
\text { Descritivo } \\
\text { Exploratório } \\
\text { com } \\
\text { abordagem } \\
\text { Quantitativa }\end{array}$ & $\begin{array}{l}\text { Investigar os fatores relacionados } \\
\text { à ocorrência de acidentes com } \\
\text { material biológico entre } 101 \\
\text { profissionais de enfermagem } \\
\text { atuantes em unidade de } \\
\text { emergência }\end{array}$ \\
\hline $\begin{array}{l}\text { RUAS, Edna de } \\
\text { Freitas Gomes } \\
\text { et al. }\end{array}$ & 2012 & 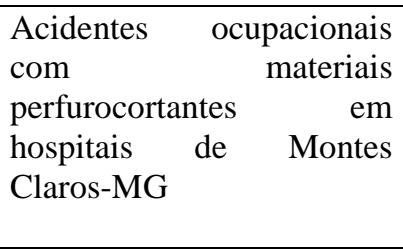 & $\begin{array}{l}\text { Pesquisa } \\
\text { Descritiva } \\
\text { Retrospectiva } \\
\text { com } \\
\text { abordagem } \\
\text { quantitativa }\end{array}$ & $\begin{array}{llr}\text { Descrever } & \text { e caracterizar } & \text { os } \\
\text { acidentes } & \text { ocupacionais } & \text { com } \\
\text { material } & \text { perfurocortante } & \text { e } \\
\text { exposição. } & & \end{array}$ \\
\hline
\end{tabular}

Fonte: Dados da pesquisa, 2015.

A pesquisa com delineamento transversal foi encontrada em 2 artigos que segundo Rodrigues (2007), tem como finalidade descrever características pessoais de determinada população junto com sua história. Enquanto na abordagem da pesquisa a maior prevalência com oito artigos foram os estudos quantitativos em detrimento da qualitativa com apenas dois. Para Oliveira (2013), a pesquisa quantitativa tem como finalidade a objetividade usando técnicas estatísticas para vinculação de dados que são devidamente mensuráveis.

$\mathrm{O}$ tipo de pesquisa descritiva exploratória foi evidenciada em 8 artigos, tendo como objetivo a utilização de técnicas padronizadas de coleta de dados a partir de uma observação sistemática. E a exploratória tem como finalidade proporcionar maior familiaridade com o problema. (RODRIGUES, 2007).

Basicamente em todos os artigos selecionados, os objetivos são parecidos e tratam de investigar a relação dos riscos biológicos com os acidentes de trabalho. A apresentação dos objetivos e dos resultados obtidos nos estudos foi feita de forma descritiva.

Em vários estudos constatou-se a resistência dos profissionais ao uso de EPI 
(equipamento de proteção individual) contribuindo para uma maior vulnerabilidade aos riscos biológicos. Contudo, é importante salientar que o emprego de práticas seguras e o uso de equipamentos de proteção adequados reduzem significativamente o risco de acidente ocupacional. Fazendo-se necessária também à conscientização dos profissionais para utilização de técnicas assépticas, o estabelecimento de normas de conduta, além de procedimentos que garantam ao profissional e ao paciente um tratamento sem risco de contaminação (SIMÃO et al., 2010).

O estudo de Guilard (2010) aponta que $30 \%$ dos acidentados não haviam sido vacinados contra Hepatite $B$, o que reforça a necessidade de oferecer a vacinação para todos os profissionais de saúde da instituição, pois constantemente estão vulneráveis por vários riscos biológicos e consequentemente a se contaminarem. Portanto, como medida preventiva, todo trabalhador de serviços de saúde deve receber, gratuitamente, imunização ativa contra tétano, difteria, hepatite B e os estabelecidos no Programa de Controle Médico de Saúde Ocupacional (SIMÃO et al., 2010).

Sendo assim, no estudo de Machado (2011) traz algumas medidas que podem contribuir para a prevenção no atendimento mais rápido e eficaz aos profissionais acidentados, como na importância da reformulação do protocolo de encaminhamento para $\mathrm{o}$ atendimento ao acidentado e a participação efetiva dos profissionais de enfermagem. A classe mais acometida por acidentes de trabalho a matérias biológicos são os auxiliares de enfermagem pelo motivo de serem mais expostos aos riscos biológicos e por permanecer maior parte do tempo na assistência direta executando procedimentos mais invasivos, sendo com materiais perfuro cortantes mais utilizados na prática diária.

São recomendados alguns cuidados para o manuseio de materiais perfuro cortantes e com a máxima atenção durante a realização dos procedimentos, muitos profissionais da área da saúde, em especial os de enfermagem, não têm uma boa aceitação e cumprimento das normas de biossegurança, quanto maior o esclarecimento sobre o risco, adoção de medidas de proteção, menor o índice da ocorrência de doenças relacionadas ao trabalho (SOARES et al., 2013).

Várias pesquisas abordaram a educação permanente que deve ser empregada cotidianamente a fim de oferecer uma abordagem educativa capaz de modificar a realidade do trabalhador. Problematizando as situações de exposição a materiais biológicos vivenciados no cotidiano discutindo as causas de ocorrências é uma estratégia que contribui para a conscientização do risco além de levar os 
trabalhadores a identificarem os desafios pessoais (RIBEIRO et al., 2010).

Não se limitando apenas a questão física, alguns estudos também abordaram temas que envolvem os sentimentos dos profissionais de enfermagem. Como no estudo de Araújo et al (2012) apresenta os emoção vivenciados pelos trabalhadores após a ocorrência do acidente. Havendo reações como medo, ansiedade, nervosismo, desespero e tristeza relacionados à possibilidade de contaminação pelo vírus do HIV. Ainda sobre a questão psicológica Ribeiro et al (2010) afirma que a exposição levou a reflexão sobre a situação dos acidentes e ocorreu a consciência dos riscos, revelada pelos sentimentos de alterações psicoemocionais, sociais e familiar.

Observou-se que vários autores relataram existir muitas subnotificações, junto com a ausência de programas que sensibilizem os profissionais de saúde quanto os riscos que estão opostos. $\mathrm{O}$ estudo minucioso acerca das causas dos acidentes de trabalho pode contribuir significativamente para a redução de sua ocorrência. O trabalhador, quando orientado, pode evitar as situações de risco e a gestão pode direcionar e adaptar medidas mistigatórias de risco à realidade desses profissionais. (BAKKE; ARAÚJO, 2010).

\section{CONSIDERAÇÕES FINAIS}

São diversos os riscos no qual os profissionais de Enfermagem estão expostos, sendo os mais comuns os riscos biológicos, colocando muitas vezes a saúde dos trabalhadores em perigo.

Observa-se a importância da aquisição da instituição por dispositivos mais seguros, na mudança de comportamento do funcionamento e nas falhas de suprimento da equipe, e do acompanhamento dos trabalhadores potencialmente expostos. E principalmente protocolos estabelecidos com condutas que sejam claras na empresa.

Além disso, deve ser investida numa educação permanente para seus empregadores, pois nota-se que ainda há necessidade da sensibilização acerca da importância do uso dos equipamentos de proteção individual. É de fundamental importância ações de educação permanente acerca da adoção das estratégias de biossegurança com ênfase na saúde e qualidade de vida do trabalhador, no âmbito de suas atividades laborais.

Diagnosticar e tratar trabalhadores com carga horária elevada, como dupla jornada de trabalho, de uma forma preventiva de acidentes mostra-se uma medida eficaz na redução de acidentes com material biológico, ergonômico, físico ou químico, visto que o trabalhador da Enfermagem constantemente 
assume essa jornada, sem considerar que a maior parte da equipe é composta por mulheres, mães, com falha no repouso devido às atribuições inerentes à posição de mãe e dona de casa.

A vigilância por parte da equipe de saúde do trabalhador nas empresas hospitalares precisa ser ampla visando cada situação, risco ocupacional adicionado de características pessoais se objetiva minimizar os riscos de acidentes. Medida de desestresse, ginástica laboral, dinâmicas em grupo e tentativas de conscientização tem demonstrado resultados satisfatórios na luta contra a diminuição dos acidentes de trabalho com profissionais da Enfermagem.

\section{REFERÊNCIAS}

BAKKE, H. A; ARAÚJO, N. M. C. Acidentes de trabalho com profissionais de saúde de um hospital universitário. Revista Produção. João Pessoa (PB), v. 20, n. 4. 2010.

PIRES, D. Reestruturação produtiva e trabalho em saúde no Brasil. 2 ed. São Paulo:

AnnaBlume/CNTSS; 2008.

PROCHNOW, A. et al. Acidente de Trabalho: uma revisão integrativa. Revista de Enfermagem da UFSM, 2012.

SCHMOELLERA Roseli, TRINDADE, Letícia de Lima; NEIS, Márcia Binder; Revista Gaúcha de Enfermagem; 32(2):368-37, jun.2011.tab

SOARES, L. G. et al. Percepção do risco biológico em trabalhadores de enfermagem. Cogitare enfermagem. Curitiba, v. 18, n. 1, mar. 2013.

VALIM, M. D; MARZIALE, M. H. P. Avaliação da exposição ocupacional a material biológico em serviços de saúde. Texto contexto - enfermagem. Florianópolis, v. 20, n. spe, 2011.

BRASIL. Ministério do trabalho e emprego.

Secretaria de segurança e saúde no trabalho.

Portaria $\mathrm{n}^{\circ}$ 3.214, de 08 de Junho de 1978.

Brasília 1978.

RIBEIRO, E.J.G, SHIMIZU, H.E. Acidentes de trabalho com trabalhadores de enfermagem. Rev. bras. Enferm. 2007 Set/Out;60(5):535-40

RODRIGUES, W. C. Metodologia Científica. FAETEC/IST. Paracambi, 2007.

OLIVEIRA, L. P. B. A. et al. Potencialidades e limites das abordagens de pesquisa qualitativa e quantitativa. Anais do $17^{\circ}$ Seminário Nacional de Pesquisa em Enfermagem, Natal: ABEn, 2013.

MACHADO, M.R.M; MACHADO,F.A.

Acidentes com material Biológico em Trabalhadores de Enfermagem do Hospital Geral de Palmas.Rev.bras saúde ocup, São Paulo, 2011.

RIBEIRO,L.C.M. et al. Influencia da exposição a material Biológico na adesão ao uso de equipamentos de proteção individual.Rev. Cuid Saúde, Goiania, 2010.

LIMA, L.M ; OLIVEIRA, C.C; RODROGUES, K.M.R. Exposição ocupacional por material biológico no Hospital Santa Casa de Pelotas-2004 a 2008. Rev. Esc. Anna Nery, 2011.

ARAÚJO, T.M. et al . Acidente ocupacional e contaminação pelo HIV: Sentimentos vivenciados pelos profissionais de Enfermagem. Rev. Pés. Cuid Fundam. Online, Rio de Janeiro, 2012.

MAGAGUINI, M.A.M, ROCHA, S.A, AYRES, J.A. Rev. Gaúcha Enferm, Porto Alegre(RS), 2011.

PIMENTA, F.R. et al. Atendimento e seguimento clínico especializado de profissionais de enfermagem acidentados com material biológico .Rev. Esc. Enferm USP, São Paulo, 2013.

GUILARDE, A.O. et al. Acidentes com material biológicos entre profissionais de Hospital 
Universitário em Goiânia. Rev de patologia

Brasileira. v. 39, 2010.

SIMÃO, S.A. et al. Fatores associados aos acidentes Biológicos entre profissionais de Enfermagem. Rev. Cogitare Enferm, 2010.

RUAS, E.F.G. et al Acidentes ocupacionais com materiais perfuro cortantes em Hospitais de Montes Claros- MG. Rev. Min. Enferm, 2012.

GLANCOTTI, G.M. et al. Caracterização das vítimas e dos acidentes de trabalho com materiais biológicos atendidas em um Hospital Público do Paraná. Rev. Epidemiol. Serv. Saúde, Brasília, 2014. 